\title{
Method to restore images from chaotic frequency-down-converted light using phase matching
}

\author{
Alessandra Andreoni* and Emiliano Puddu \\ Dipartimento di Fisica e Matematica and Consiglio Nazionale delle Ricerche-Istituto Nazionale per la Fisica della Materia, \\ CNR-INFM, Via Valleggio 11, 22100 Como, Italy \\ Maria Bondani \\ National Laboratory for Ultrafast and Ultraintense Optical Science, CNR_INFM, Via Valleggio 11, 22100 Como, Italy
}

(Received 18 August 2006; published 3 November 2006)

\begin{abstract}
We present an optical frequency-down-conversion process of the image of an object illuminated with chaotic light in which also the low-frequency field entering the second-order nonlinear crystal is chaotic. We show that the fulfillment of the phase-matching conditions by the chaotic interacting fields provides the rules to retrieve the object image by calculating suitable correlations of the local intensity fluctuations even if a single record of down-converted chaotic image is available.
\end{abstract}

DOI: 10.1103/PhysRevA.74.053802

PACS number(s): 42.65.Lm, 42.30.Va

\section{INTRODUCTION}

Stochastic image restoration is a challenging task with applications in many fields, such as astronomy, underwater sensing, and medical diagnostics. Specific methods have been developed over the years for each application. Only to mention the procedures that are suitable for speckle images of astronomical objects we span from 1980 to 2005, that is from the "shift and add" method of Bates and Cady [1] to the "self-deconvolving data reconstruction algorithm" of Sudo and Baba [2]. Today we know that suitable spatial intensity correlations are important ingredients of protocols for nonlocal image retrieval that work at high photon-flux densities and are either based on quantum-entangled or on classically correlated thermal light (see the review by D'Angelo and Shih [3] and other recent works [4-6]). In any of these protocols two optical arms are recognizable, in which momentum and/or position of the photons are measured, and the computation of a correlation function between these two sets of data allows retrieving the image [7].

In this paper we address the restoration of the image of an object illuminated with incoherent (pseudothermal) light that is frequency-down-converted (FDC) by using a chaotic lowfrequency input field in a $\chi^{(2)}$ nonlinear medium. Image retrieval is achieved by calculating the correlation coefficients of the local intensity fluctuations in the FDC chaotic images with the intensity fluctuations of one Fourier component of the low-frequency input field. Similar methods of spatial intensity correlation are widely adopted in scheme of image transfer, both classical and nonclassical, and at any photon flux density. Our FDC images are chaotic as the lowfrequency field entering the crystal is ideally stochastic [8], whereas the light in the two measuring arms is classically correlated as it is produced by a seeded frequency downconversion process [9]. We show that the computation of the correlation function takes advantage of the phase-matching (PM) condition in which the spatially broadband chaotic

\footnotetext{
*Corresponding author. Electronic address:
} Alessandra.Andreoni@uninsubria.it fields interact in three dimensions (3D). In fact, repeating the calculations of the correlation coefficients in parallel for many components of the Fourier transform of the lowfrequency input field yields as many spatial intensity correlation maps, in which the image is reconstructed at different positions that can be related to each other. We derive these relations from the fulfillment of the PM conditions in 3D and exploit them in new procedures for image recovery that are fast, as they work with correlation maps involving ensemble averages on relatively small numbers of chaotic-image records, and allow nonlocal image recovery. Our results could lead to protocols for secure and fast transmission of images.

\section{EXPERIMENT}

With the experimental setup that is sketched in Fig. 1, we realize the frequency down-conversion at frequency degeneracy of the object field produced by an amplitude modulating mask (noncollinear type-I interaction in a $\beta-\mathrm{BaB}_{2} \mathrm{O}_{4}$ crystal, BBO I, $\left.\vartheta_{\text {cut }}=32^{\circ}\right)$. The mask, which is shown in the photograph in Fig. 1(a), is back irradiated by a ns-pulsed field at $\omega_{3}=\omega_{1}+\omega_{2}=2 \omega$ [second harmonic output $(\mathrm{SH})$ of a $Q$-switched Nd:YAG laser, $10 \mathrm{~Hz}$ repetition rate]. All other equipment and optical components are as in Ref. [5]. The input field at $\omega_{1}$ is made pseudothermal by a moving groundglass plate (D in Fig. 1) [10]. As in previous works of ours $[5,11]$, we record the local intensity distributions, $I_{2}\left(i^{\prime}, j^{\prime}\right)$, of single-shot FDC chaotic images in the "image plane, $\mathrm{P}_{\mathrm{IM}}$ " by a charge-coupled device (CCD) camera, which is synchronized with the $\mathrm{Nd}$ laser, and correlate the fluctuations $\Delta I_{2}\left(i^{\prime}, j^{\prime}\right)$ with those, $\Delta I_{1}(i, j)$, of the content of the CCD pixel $(i, j)$ recording the Fourier transform ("Fourier transform plane, $\mathrm{P}_{\mathrm{FT}}$ ") of the $\omega_{1}$ field at the same shot. Such a partition of the CCD sensor is sketched in Fig. 1(b). We consider two distinct cases for the field at $\omega_{3}$ that impinges onto the object mask: (i) the field is provided by the $\mathrm{SH}$ output of the laser as it is; and (ii) the field is made incoherent by further inserting the moving ground glass plate $\mathrm{D}^{\prime}$ on the SH output as shown in Fig. 1. Actually, in both cases, we calculate 


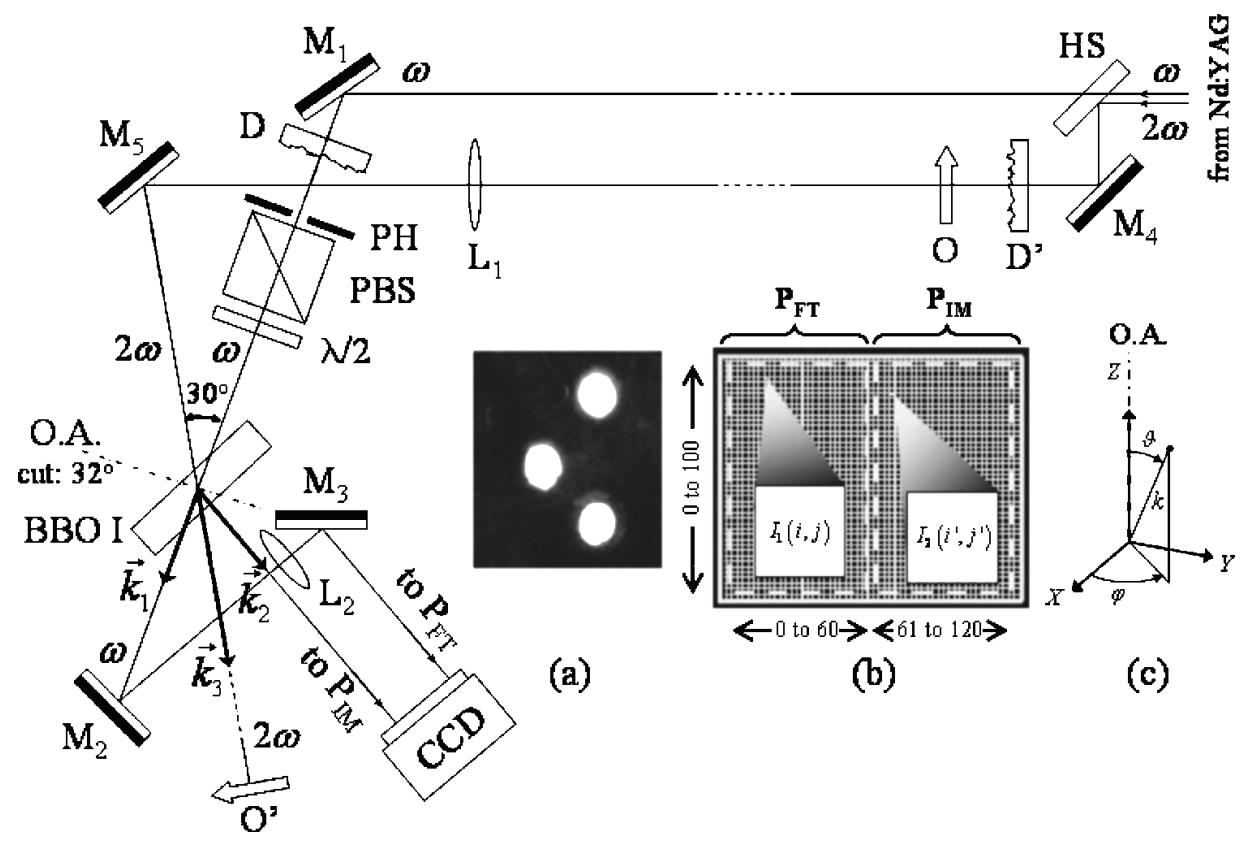

FIG. 1. Schematic of the experimental setup. Lens $\mathrm{L}_{1}$ produces the 1-to-1 virtual image $\mathrm{O}^{\prime}$ of the object $\mathrm{O}$, which is frequency downconverted into a real image on focus at plane $\mathrm{P}_{\mathrm{IM}}$, right-hand side part of the CCD sensor. The spatial spectrum of the low-frequency input beam is detected on plane $\mathrm{P}_{\mathrm{FT}}$, which is the focal plane of lens $\mathrm{L}_{2}(f=15 \mathrm{~cm})$. The distance of BBO I to $\mathrm{O}^{\prime}$ is $40 \mathrm{~cm}$, that to $\mathrm{P}_{\mathrm{IM}}$ is $20 \mathrm{~cm}$ (Ref. [15]). (a) Microphotograph of object O, an opaque mask with three holes of $\sim 256 \mu \mathrm{m}$ diameter. (b) Partition of the CCD sensor (pixel size: $16 \mu \mathrm{m} \times 16 \mu \mathrm{m}$ ). (c) Crystal reference frame with $Z$ parallel to the optical axis, O.A., horizontal in the real setup of main panel.

$$
\begin{aligned}
G_{i, j}\left(i^{\prime}, j^{\prime}\right) & =\left\langle\Delta I_{1}(i, j) \Delta I_{2}\left(i^{\prime}, j^{\prime}\right)\right\rangle \\
& \equiv\left\langle I_{1}(i, j) I_{2}\left(i^{\prime}, j^{\prime}\right)\right\rangle-\left\langle I_{1}(i, j)\right\rangle\left\langle I_{2}\left(i^{\prime}, j^{\prime}\right)\right\rangle,
\end{aligned}
$$

which, when $I_{1}(i, j)$ represents the intensity carried by a single spatial Fourier component of the low-frequency input field, is known to reproduce the FDC image generated by the interaction of this component (wave vector $\mathbf{k}_{1}$ ) with the object field at $\omega_{3}$ [wave vector(s) $\mathbf{k}_{3}$ ]. Obviously this holds for wave vectors fulfilling the PM condition with the tolerance set by the bandwidth of the interaction. As the interaction is most conveniently described in the crystal reference frame $(X, Y, Z)$, in which the BBO I optical axis is the $Z$ axis [see panel (c) of Fig. 1], it is worth mentioning that in our experiment the $(Y, Z)$ plane is horizontal, while the CCD sensor of panel (b) lies in a vertical plane. Beams, mirrors, and CCD are oriented in such a way that, when the diffusing plates D and $\mathrm{D}^{\prime}$ are removed, both $\mathbf{k}_{1}$ and $\mathbf{k}_{2}$ get the sensor at normal incidence.

The experimental results [i.e., thousands of $I_{1}(i, j)$ and $I_{2}\left(i^{\prime}, j^{\prime}\right)$ intensity maps recorded for as many single shots] lead to $G_{i, j}\left(i^{\prime}, j^{\prime}\right)$ spatial intensity correlation maps averaged over 1000 repetitions such as those displayed in Figs. 2(i) and 2(ii) for nonchaotic and chaotic field at $2 \omega$, respectively. In the two panels of Fig. 2, the maps are organized in columns and rows according to the values of $i$ and $j$. Each map, framed in gray, displays $60 \times 100$ (i.e., $i^{\prime} \times j^{\prime}$ ) correlation coefficients. Comparing panels (i) and (ii) provides evidence that, in case (ii), the $G_{i, j}\left(i^{\prime}, j^{\prime}\right)$ maps allow image retrieval for $(i, j)$ pixels that cover a region in the $\mathrm{P}_{\mathrm{FT}}$ plane that is much wider than that in case (i): in the latter case we find an image of good quality virtually only for $i=57, j=95$; in case (ii) all $G_{i, j}\left(i^{\prime}, j^{\prime}\right)$ maps calculated for $i$ between 28 and 43 and for $j$ between 36 and 63 show clearly recognizable images. In Fig. 2(ii) a subset of these maps is displayed. Moreover, once we fixed the $(i, j)$ pixel, we obtain virtually iden- (i)

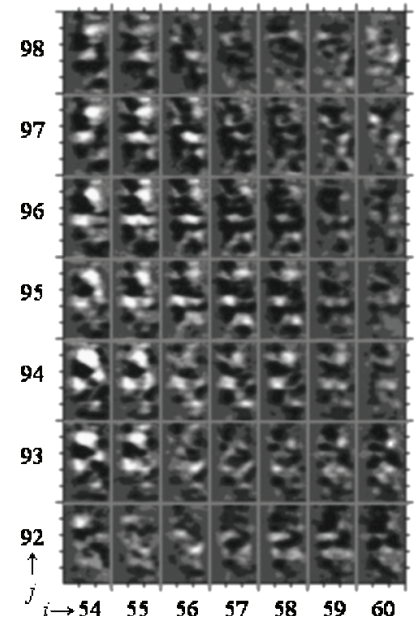

(ii)

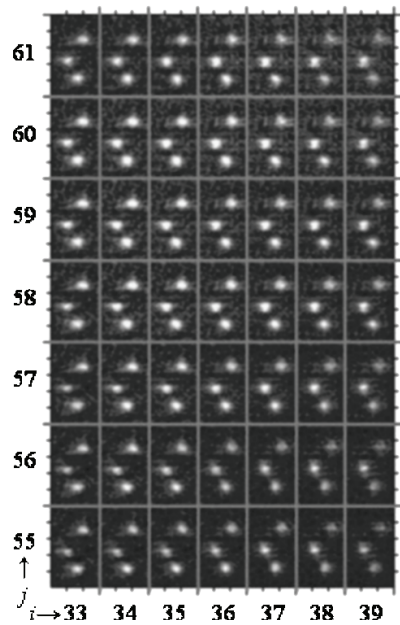

FIG. 2. Collections of $G_{i, j}\left(i^{\prime}, j^{\prime}\right)$ spatial intensity correlation maps averaged over 1000 single-shot repetitions (namely, from the 10 000th to the 10999 th) of the 20000 recorded frames. The maps in the two panels refer to (i) nonchaotic and (ii) chaotic fields at $2 \omega$. The maps are organized in columns and rows according to the values of $i$ and $j$, respectively. Each map, framed in gray, displays $60 \times 100$ (i.e., $\left.i^{\prime} \times j^{\prime}\right)$ correlation coefficients. 
tical $G_{i, j}\left(i^{\prime}, j^{\prime}\right)$ maps by using any thousand of the recorded frames to calculate the ensemble average in Eq. (1). An effect that cannot be perceived in Fig. 2(ii) is that the retrieved images undergo regular shifts in the plane $\left(i^{\prime}, j^{\prime}\right)$ when either $i$ or $j$ is changed in regular steps.

\section{THEORETICAL MODEL}

To explain these results we first demonstrate that, by working with a diffused high-frequency light in the presence of a diffused low-frequency input field, as in case (ii), we actually have difference-frequency light that reaches $\mathrm{P}_{\mathrm{IM}}$ at any position being generated in condition of PM.

In the crystal reference frame $(X, Y, Z)$ we consider three phase matched wave vectors, $\mathbf{k}_{1}, \mathbf{k}_{2}$, and $\mathbf{k}_{3}$, generally lying in a plane not containing the $Z$ axis. For these wave vectors, which we write in the spherical coordinates of Fig. 1(c),

$$
\begin{aligned}
\mathbf{k}_{n}= & k_{n} \sin \vartheta_{n} \cos \varphi_{n} \hat{X}+k_{n} \sin \vartheta_{n} \sin \varphi_{n} \hat{Y}+k_{n} \cos \vartheta_{n} \hat{Z} \\
& (n=1,2,3),
\end{aligned}
$$

the PM relation, $\mathbf{k}_{1}+\mathbf{k}_{2}=\mathbf{k}_{3}$, translates into

$$
\begin{gathered}
k_{3} \sin \vartheta_{3} \cos \varphi_{3}=k_{1} \sin \vartheta_{1} \cos \varphi_{1}+k_{2} \sin \vartheta_{2} \cos \varphi_{2}, \\
k_{3} \sin \vartheta_{3} \sin \varphi_{3}=k_{1} \sin \vartheta_{1} \sin \varphi_{1}+k_{2} \sin \vartheta_{2} \sin \varphi_{2}, \\
k_{3} \cos \vartheta_{3}=k_{1} \cos \vartheta_{1}+k_{2} \cos \vartheta_{2},
\end{gathered}
$$

and, after simple manipulations, into

$$
\begin{aligned}
k_{3}^{2}= & k_{1}^{2}+k_{2}^{2}+2 k_{1} k_{2}\left[\sin \vartheta_{1} \sin \vartheta_{2} \cos \left(\varphi_{1}-\varphi_{2}\right)\right. \\
& \left.+\cos \vartheta_{1} \cos \vartheta_{2}\right], \\
k_{2}^{2}= & k_{3}^{2}+k_{1}^{2}-2 k_{1} k_{3}\left[\sin \vartheta_{1} \sin \vartheta_{3} \cos \left(\varphi_{1}-\varphi_{3}\right)\right. \\
& \left.+\cos \vartheta_{1} \cos \vartheta_{3}\right], \\
k_{1}^{2}= & k_{3}^{2}+k_{2}^{2}-2 k_{2} k_{3}\left[\sin \vartheta_{2} \sin \vartheta_{3} \cos \left(\varphi_{3}-\varphi_{2}\right)\right. \\
& \left.+\cos \vartheta_{2} \cos \vartheta_{3}\right] .
\end{aligned}
$$

Writing the PM condition in the form of Eq. (4) emphasizes the invariance of PM for rotations about the optical axis $Z$, in agreement with the rotational symmetry of the $k$ surfaces of our uniaxial crystal [12]. Moreover, being $k_{1}=k_{2} \equiv k=k_{o}(\omega)$ for a type I interaction at frequency degeneracy, Eqs. (4) further simplify to

$$
\begin{gathered}
\frac{k_{3}^{2}}{2 k^{2}}=\left[1+\sin \vartheta_{1} \sin \vartheta_{2} \cos \left(\varphi_{1}-\varphi_{2}\right)+\cos \vartheta_{1} \cos \vartheta_{2}\right], \\
\frac{k_{3}}{2 k}=\sin \vartheta_{1} \sin \vartheta_{3} \cos \left(\varphi_{1}-\varphi_{3}\right)+\cos \vartheta_{1} \cos \vartheta_{3}, \\
\frac{k_{3}}{2 k}=\sin \vartheta_{2} \sin \vartheta_{3} \cos \left(\varphi_{3}-\varphi_{2}\right)+\cos \vartheta_{2} \cos \vartheta_{3},
\end{gathered}
$$

in which $[12,13]$

$$
k_{3}=\left[\frac{\sin ^{2} \vartheta_{3}}{k_{e}^{2}(2 \omega)}+\frac{\cos ^{2} \vartheta_{3}}{k_{o}^{2}(2 \omega)}\right]^{-1 / 2} .
$$

If we consider a particular $\mathbf{k}_{2}=\mathbf{k}_{2}\left(k, \vartheta_{2}, \varphi_{2}\right)$ that brings intensity to the pixels of $\mathrm{P}_{\mathrm{IM}}$, we observe that, for $\boldsymbol{\vartheta}_{2}>\vartheta_{3 c}$, where $\vartheta_{3 c}$ corresponds to collinear PM, according to Eqs. (5) many pairs of $\mathbf{k}_{1}$ and $\mathbf{k}_{3}$ wave vectors give rise to phasematched interactions with the chosen $\mathbf{k}_{2}$. In fact, with the definitions

$$
Y_{r}=\frac{k_{3}}{2 k \sin \vartheta_{2}}\left(k_{3}-2 k \cos \vartheta_{2} \cos \vartheta_{3}\right)
$$

and

$$
r=\left[k^{2}-\left(k_{3} \cos \vartheta_{3}-k \cos \vartheta_{2}\right)^{2}\right]^{1 / 2},
$$

the wave vectors

$$
\begin{gathered}
\mathbf{k}_{1}=\left(k, \sin ^{-1}(r / k), \varphi_{2} \pm \cos ^{-1}\left[\left(Y_{r}-k \sin \vartheta_{2}\right) / r\right]\right), \\
\mathbf{k}_{2}=\left(k, \vartheta_{2}, \varphi_{2}\right), \\
\mathbf{k}_{3}=\left(k_{3}\left(\vartheta_{3}\right), \vartheta_{3}, \varphi_{2} \pm \cos ^{-1}\left\{Y_{r} /\left[k_{3}\left(\vartheta_{3}\right) \sin \vartheta_{3}\right]\right\}\right),
\end{gathered}
$$

which are expressed in terms of the independent variables $\vartheta_{2}, \varphi_{2}$, and $\vartheta_{3}$, fulfill the PM conditions.

According to Eqs. (9) many pairs of $\mathbf{k}_{1}$ and $\mathbf{k}_{3}$ wave vectors give rise to phase-matched interactions with any $\mathbf{k}_{2}$ bringing light to $\mathrm{P}_{\mathrm{IM}}$. For instance, in a plane containing the optical axis, that is for $\varphi_{1}=\varphi_{2}=\varphi_{3}$, say $(Y, Z)$ without loss of generality as in Fig. 3(a), we find two pairs of $\mathbf{k}_{3}$ and $\mathbf{k}_{1}$ which, together with the same $\mathbf{k}_{2}$, fulfill Eqs. (5). In fact, according to Eq. (6) and to the last one of Eqs. (5), it should be $\quad\left\{2 k\left[\left(\sin ^{2} \vartheta_{3} / k_{e}^{2}\right)+\left(\cos ^{2} \vartheta_{3} / k_{o}^{2}\right)\right]^{1 / 2}\right\}^{-1}=\cos \left(\vartheta_{3}-\vartheta_{2}\right) \quad$ to achieve PM in this plane. In Fig. 3(c) we plot the first and second members of this equation as functions of $\vartheta_{3}$, for $\vartheta_{2}$ constant. For the open-dot curve we choose $\vartheta_{2}=34^{\circ}$, that is the experimental value (see $\vartheta_{\text {cut }}=32^{\circ}$ and $\vartheta_{1}^{\text {ext }}-\vartheta_{3}^{\text {ext }}=30^{\circ}$ in the main panel of Fig. 1 and the BBO data of Ref. [13]). The $\mathbf{k}_{3}$ wave vectors at the angles marked as $\vartheta_{3 a}$ and $\vartheta_{3 b}$ in Fig. 3(c) and, correspondingly, the $\mathbf{k}_{1}$ wave vectors with $\vartheta_{1 a, b}=2 \vartheta_{3 a, b}-\vartheta_{2}$ in Fig. 3(a) ensure noncollinear PM. On the contrary, Fig. 3(c) shows that any $\mathbf{k}_{3}$ with an intermediate $\vartheta_{3}$ value, say $\vartheta_{3 i}$ with $\vartheta_{3 a}<\vartheta_{3 i}<\vartheta_{3 b}$, yields $\left\{2 k\left[\left(\sin ^{2} \vartheta_{3} / k_{e}^{2}\right)+\left(\cos ^{2} \vartheta_{3} / k_{o}^{2}\right)\right]^{1 / 2}\right\}^{-1}<\cos \left(\vartheta_{3 i}-\vartheta_{2}\right)$ in agreement with the existence of two $\varphi_{3}$ values, $\varphi_{3 \pm}$ $=\pi / 2 \pm \cos ^{-1}\left\{Y_{r} /\left[k_{3}\left(\vartheta_{3 i}\right) \sin \vartheta_{3 i}\right]\right\}$ provided by the last of Eqs. (9). The projections of these $\mathbf{k}_{1}$ and $\mathbf{k}_{3}$ phase-matched wave vectors are shown in Fig. 3(b), in which the quantity $Y_{r}$ is indicated and $r$ is the radius of $\sigma_{1}^{\prime}$.

From Eqs. (9) we can also obtain the rules governing the shifts in plane $\mathrm{P}_{\mathrm{IM}}$ among images recovered by correlation coefficients $G_{i, j}\left(i^{\prime}, j^{\prime}\right)$ calculated for different $(i, j)$. We first transpose the results in Eqs. (9) from the crystal reference frame $(X, Y, Z)$ into the laboratory reference frame $(x, y, z)$ depicted in Fig. 3(d) in which $x \equiv X$, plane $(y, z)$, which lies horizontally, contains the optical axis $Z$, and the $z$ axis is along the normal to the crystal entrance face. If $\eta_{V, n}$ and $\eta_{H, n}$ denote the latitude and longitude angles in the $(x, y, z)$ frame of the wave vectors out of the crystal, we have 

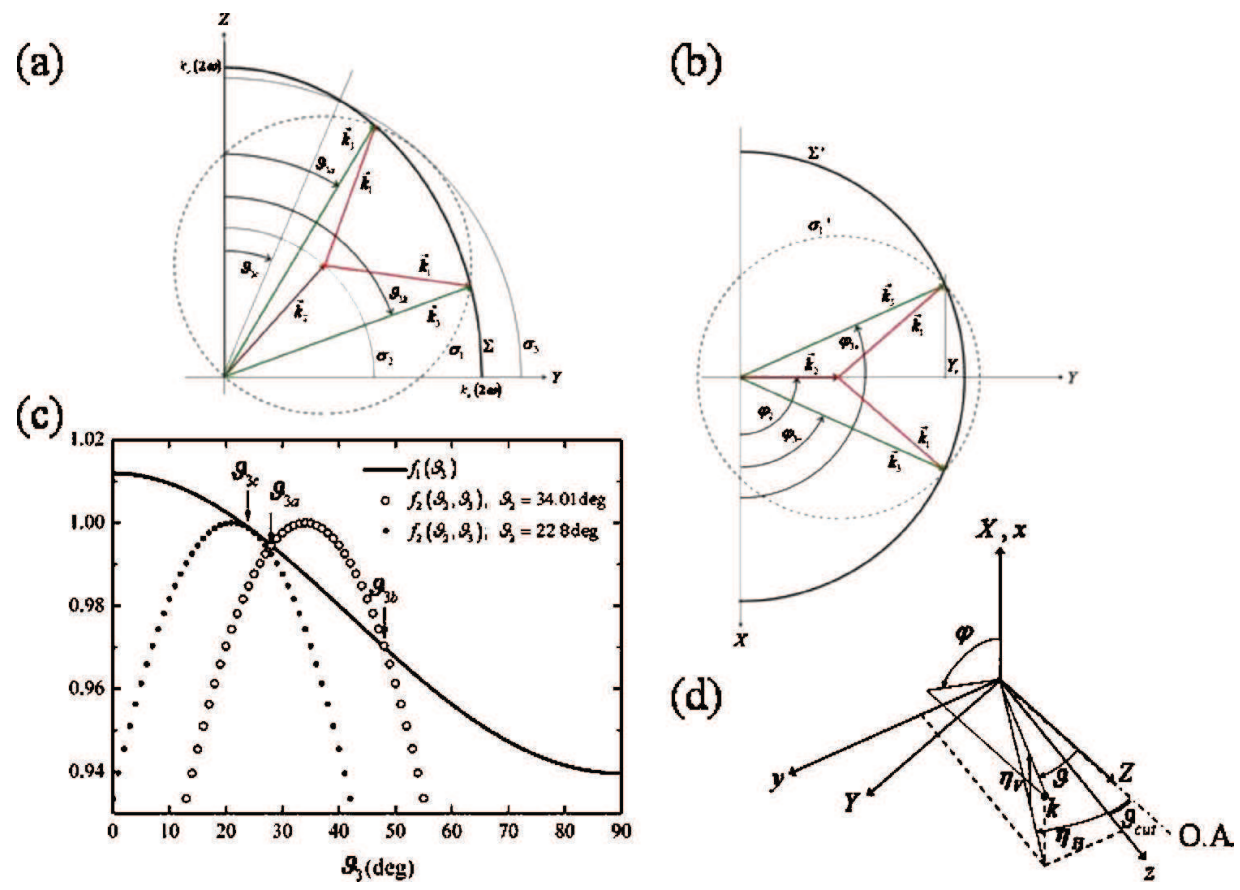

FIG. 3. (Color online) (a) Intersections of $k$ surfaces ( $\Sigma$ ellipsoid, $\sigma$ 's spheres) with the $(Y, Z)$ plane and phase-matched wave vectors lying on the $(Y, Z)$ plane, i.e., $\varphi_{1}=\varphi_{2}=\varphi_{3}=\pi / 2$. Collinear phase matching at $\vartheta_{3 c}$. $\mathbf{k}_{2}$ is at any fixed $\vartheta_{2}>\vartheta_{3 c}$. The two pairs of $\mathbf{k}_{3}$ and $\mathbf{k}_{1}$ wave vectors are phase matched to $\mathbf{k}_{2}$, being the values of angles $\vartheta_{3 a}$ and $\vartheta_{3 b}$ those in (d). Radii of $\sigma_{1}$ and $\sigma_{2}: k_{o}(\omega)$. Radius of $\sigma_{3}: 2 k_{o}(\omega)$. (b) Projections of phase-matched wave vectors on the $(X, Y)$ plane. $\mathbf{k}_{2}$ is the same wave vector, $\mathbf{k}_{2}\left(\vartheta_{2}\right)$, as in (a), while both $\mathbf{k}_{3}$ wave vectors are at angle $\vartheta_{3 i}$, being $\vartheta_{3 a}<\vartheta_{3 i}<\vartheta_{3 b}$. The two pairs of $\mathbf{k}_{3}$ and $\mathbf{k}_{1}$ wave vectors phase matched to $\mathbf{k}_{2}$ are symmetrical with respect to the $(Y, Z)$ plane. Traces $\Sigma^{\prime}$ and $\sigma_{1}^{\prime}$ : projections of the $\Sigma$ and $\sigma$ intersections with plane $Z=k_{3}\left(\vartheta_{3 i}\right) \cos \vartheta_{3 i}$. The radii are $R=k_{3}\left(\vartheta_{3 i}\right) \sin \vartheta_{3 i}$ for $\Sigma^{\prime}$ and $r=\left\{k_{o}^{2}(\omega)-\left[k_{3}\left(\vartheta_{3 i}\right) \cos \vartheta_{3 i}-k_{o}(\omega) \cos \vartheta_{2}\right]^{2}\right\}^{1 / 2}$ for $\sigma_{1}^{\prime}$. (c) Plots of the functions $f_{1}=\left\{2 k\left[\left(\sin ^{2} \vartheta_{3} / k_{e}^{2}\right)+\left(\cos ^{2} \vartheta_{3} / k_{0}^{2}\right)\right]^{1 / 2}\right\}^{-1}$ (full line) and $f_{2}=\cos \left(\vartheta_{3}-\vartheta_{2}\right)$, for $\vartheta_{2}=34^{\circ}$ (open dots). The values $\vartheta_{3 a} \cong 28^{\circ}$ and $\vartheta_{3 b} \cong 48^{\circ}$ of the intersections correspond to noncollinear phase matching $\left(\vartheta_{1 a} \cong 22^{\circ}\right.$ and $\left.\vartheta_{1 b} \cong 62^{\circ}\right)$. Full dots: as above for $\vartheta_{2}=22.8^{\circ}=\vartheta_{3 c}$ (collinear phase matching). (d) Crystal reference frame $(X, Y, Z)$ and laboratory reference frame $(x, y, z)$.

$$
\eta_{V, n}=\sin ^{-1}\left(n_{n} \sin \vartheta_{n} \cos \varphi_{n}\right)
$$

and

$$
\begin{aligned}
\eta_{H, n}= & \sin ^{-1}\left\{\frac{n_{n} \sqrt{1-\sin ^{2} \vartheta_{n} \cos ^{2} \varphi_{n}}}{\sqrt{1-n_{n}^{2} \sin ^{2} \vartheta_{n} \cos ^{2} \varphi_{n}}}\right. \\
& \left.\times \sin \left[\left(\cos ^{-1} \frac{\cos \vartheta_{n}}{\sqrt{1-\sin ^{2} \vartheta_{n} \cos ^{2} \varphi_{n}}}\right)-\vartheta_{\text {cut }}\right]\right\},
\end{aligned}
$$

in which $n_{n}$ are the refractive indices $n_{1}=n_{2}=n_{o}(\omega)$ and $n_{3}$ $=n_{3}(2 \omega)$. Upon writing Eqs. (10) and (11) in terms of the independent variables $\vartheta_{1}, \varphi_{1}$, and $\vartheta_{3}$ we calculate the expressions of $d \eta_{V, n}$ and $d \eta_{H, n}$ with $n=1,2$ for constant $\vartheta_{3}$. The results for $\varphi_{n} \cong \pi / 2$ as in our experimental condition are

$$
\begin{gathered}
d \eta_{V, 1} \cong-n_{1} \sin \vartheta_{1} d \varphi_{1}, \\
d \eta_{V, 2} \cong-d \eta_{V, 1},
\end{gathered}
$$

and

$$
d \eta_{H, 1} \cong \frac{n_{1} \cos \left(\vartheta_{1}-\vartheta_{\text {cut }}\right)}{\sqrt{1-n_{1}^{2} \sin ^{2}\left(\vartheta_{1}-\vartheta_{\text {cut }}\right)}} d \vartheta_{1}
$$

$$
\begin{aligned}
d \eta_{H, 2} \cong & -n_{1} \sin \vartheta_{1} \frac{\cos \left(\vartheta_{2}-\vartheta_{\text {cut }}\right)}{\cos \left(\vartheta_{1}-\vartheta_{\text {cut }}\right)} \\
& \times \frac{\sqrt{1-n_{1}^{2} \sin ^{2}\left(\vartheta_{1}-\vartheta_{\text {cut }}\right)}}{\sqrt{1-n_{2}^{2} \sin ^{2}\left(\vartheta_{2}-\vartheta_{\text {cut }}\right)}} d \eta_{H, 1} .
\end{aligned}
$$

Since maps belonging to a row in Fig. 2(ii), i.e., fixed $j$ and varying $i$ values, correspond to $d \eta_{V, 1}=0$, the images retrieved by these maps are produced by $\mathbf{k}_{1}$ wave vectors with equal $\varphi_{1}$ values as, according to the first of Eqs. (12), $d \varphi_{1}$ $=0$ for any $\vartheta_{1}$. As also $d \eta_{V, 2}=0$, they are displayed at equal vertical positions on plane $\left(i^{\prime}, j^{\prime}\right)$. Moreover Eqs. (12) provide one-to-one links $\Delta j^{\prime} \leftrightarrow \Delta j \leftrightarrow d \varphi_{1}$ [14]. On the other hand, Eqs. (13) tell that maps belonging to a column in Fig. 2(ii), i.e., fixed $i$ and varying $j$ values, which correspond to $d \eta_{H, 1}=0$, refer to images retrieved by correlations with $\mathbf{k}_{1}$ wave vectors with constant $\vartheta_{1}$ angles. Also, for the horizontal direction $d \eta_{H, 2}=0$, but the links $\Delta i^{\prime} \leftrightarrow \Delta i \leftrightarrow d \vartheta_{1}$ are more complex.

\section{DISCUSSION}

On the grounds of Eq. (9) we can explain the result in Fig. 2(ii) as compared to that in Fig. 2(i). In case (ii) the $I_{2}\left(i^{\prime}, j^{\prime}\right)$ 


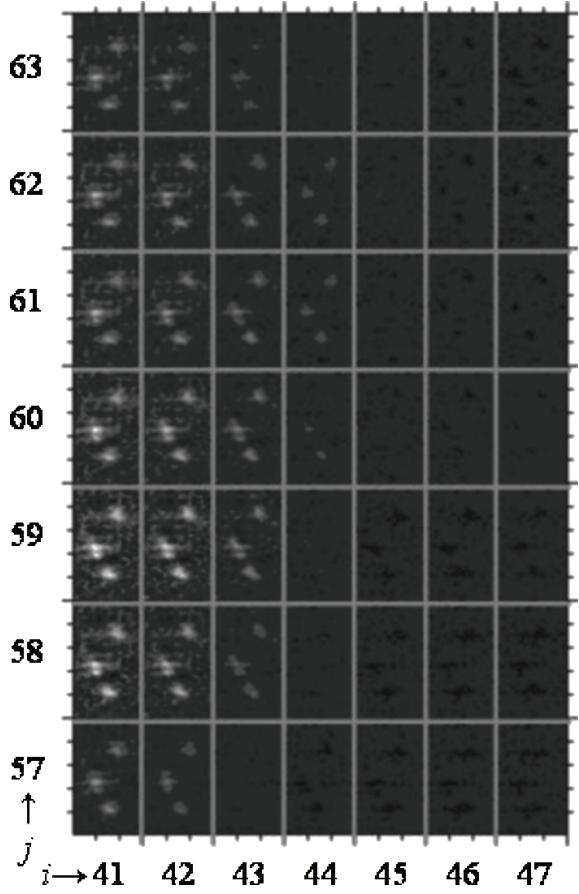

FIG. 4. Collection of $G_{i, j}\left(i^{\prime}, j^{\prime}\right)$ spatial intensity correlation maps averaged over 1000 recorded frames (namely, from the 5000th to 5999th). Each map displays $60 \times 100$ (i.e., $\left.i^{\prime} \times j^{\prime}\right)$ correlation coefficients.

values exhibit stronger correlations with the $I_{1}(i, j)$ values for any $(i, j)$ that allows PM because any of the $\mathbf{k}_{2}$ components of the field generated at the difference frequency participates to phase-matched interactions with more pairs of $\mathbf{k}_{1}$ and $\mathbf{k}_{3}$ wave vectors. Thus, for a given far-field distribution of the low-frequency input field $I_{1}(i, j)$ measured on plane $\mathrm{P}_{\mathrm{FT}}$, the broader the angular spectrum of the high-frequency field $\left(\mathbf{k}_{3}\right.$ wave vectors), the better we recover the image by using Eq. (1). In our experiment the $\eta_{V, n}$ and $\eta_{H, n}$ angles are centered at $0^{\circ}$ and, as the shift by one pixel on plane $\mathrm{P}_{\mathrm{FT}}$ corresponds to $p / f=6.1 \times 10^{-3} \mathrm{deg}$, where $f$ is the focal length of lens $\mathrm{L}_{2}$ and $p$ is the linear size of the pixels (see Fig. 1), the full angular spreads of $\mathbf{k}_{1}$ wave vectors that we can use to recover the image information carried by the $\mathbf{k}_{2}$ wave vectors are $\Delta \eta_{V, 1}=\Delta j(p / f)=0.165^{\circ} \quad(j=36$ to 63 , see Sec. II $)$, $\Delta \eta_{H, 1}=\Delta i(p / f)=0.092^{\circ} \quad(i=28$ to 43$)$. These $\Delta \eta_{V, 1}$ and $\Delta \eta_{H, 1}$ values should be compared with the $6.1 \times 10^{-3} \mathrm{deg}$ covered by the single pixel $(57,95)$ for which the image is recovered in Fig. 2(i).

According to Fig. 3(c), the broad angular spectrum that leads to image recovery in case (ii) should present sharp edges in correspondence with the $\vartheta_{1 a}$ and $\vartheta_{1 b}$ values calculated from those of $\vartheta_{3 a}$ and $\vartheta_{3 b}$. Since, with the $2 \omega$ and $\omega$ beams entering BBO I as shown in the main panel of Fig. 1, we operated with angles in the vicinity of $\vartheta_{1 b}$ and $\vartheta_{3 b}$, we could observe this effect as shown in Fig. 4. Here we plot a subset of $G_{i, j}\left(i^{\prime}, j^{\prime}\right)$ spatial intensity correlation maps (averaged over 1000 repetitions, incidentally from the 5000th to 5999th) organized as those in Fig. 2 for $i$ comprised between 41 and 47 ( $j=57$ to 63$)$ and notice that the image is recovered only for $i$ up to 43. Nothing similar can be observed in the vertical direction (index $j$, angle $\varphi_{1}$ ), where the images are recovered over a broader range of $\varphi_{1}$ angles $\left(\Delta \eta_{V, 1}\right.$ $\left.=0.165^{\circ}\right)$ and the disappearance of the image occurs very smoothly.

We now verify that the shifts between the images reconstructed by $G_{i, j}\left(i^{\prime}, j^{\prime}\right)$ and $G_{i+\Delta i, j+\Delta j}\left(i^{\prime}, j^{\prime}\right)$ agree with Eqs. (12) and (13). In the experiment we measure $d \eta_{V, 1}$ and $d \eta_{V, 2}$ as $\Delta j p / f$ and $\Delta j^{\prime} p / \ell$, where $\ell$ is the BBO I distance from the CCD sensor while $f$ and $p$ were already defined. Similarly we measure $d \eta_{H, 1}$ and $d \eta_{H, 2}$ as $\Delta i p / f$ and $\Delta i^{\prime} p / \ell$. From Eqs. (12) we get $\Delta j^{\prime}=-\Delta j \ell / f$, while from Eqs. (13) we get $\Delta i^{\prime} \cong-0.949 \Delta i \ell / f$, being $\ell / f=4 / 3, \quad n_{1}=n_{2}$ $=1.65451, \vartheta_{1}=62^{\circ}, \vartheta_{2}=34^{\circ}$, and $\vartheta_{\text {cut }}=32^{\circ}$, in our case. Thus, we expect that any image recovered by $G_{i, j}\left(i^{\prime}, j^{\prime}\right)$ is also recovered by $G_{i+\Delta i, j+\Delta j}\left(i^{\prime}+\Delta i^{\prime}, j^{\prime}+\Delta j^{\prime}\right)$, where the shifts are such that $3 \Delta j^{\prime}=-4 \Delta j$ and $4 \Delta i^{\prime}=-5 \Delta i$, being $5 / 4$ our best approximation of $0.949 \ell / f$. We have chosen an alternative method to demonstrate the correctness of these shifts, which is less trivial but useful for the applications. We consider four series of $G_{i+\Delta i, j}\left(i^{\prime}, j^{\prime}\right)$ for $\Delta i=0,4,8, \ldots$ starting from $i, i+1, i+2, i+3$ and, for each $i$, three series of $G_{i, j+\Delta j}\left(i^{\prime}, j^{\prime}\right)$ for $\Delta j=0,3,6, \ldots$ starting from $j, j+1, j+2$; summing the contents of these maps according to the rule $G_{i, j}\left(i^{\prime}, j^{\prime}\right)+G_{i+4, j}\left(i^{\prime}+5, j^{\prime}\right)+G_{i+8, j}\left(i^{\prime}+10, j^{\prime}\right)+\ldots$ produces four columns of maps, $S_{i, j}\left(i^{\prime}, j^{\prime}\right), S_{i+1, j}\left(i^{\prime}, j^{\prime}\right), S_{i+2, j}\left(i^{\prime}, j^{\prime}\right)$, $S_{i+3, j}\left(i^{\prime}, j^{\prime}\right)$ that we number by the values of $i, i+1, i+2, i$ +3 . If we further sum according to the rule $S_{i, j}\left(i^{\prime}, j^{\prime}\right)$ $+S_{i+1, j}\left(i^{\prime}+1, j^{\prime}\right)+S_{i+2, j}\left(i^{\prime}+2, j^{\prime}\right)+S_{i+3, j}\left(i^{\prime}+3, j^{\prime}\right) \equiv S_{j}\left(i^{\prime}, j^{\prime}\right)$, we find a single column of maps that we could compare to the $i$ th column in Fig. 2(ii). By applying the same procedure to the $S_{j}\left(i^{\prime}, j^{\prime}\right)$ maps in the $j$ direction, that is by calculating the three sums $U_{j}\left(i^{\prime}, j^{\prime}\right) \equiv S_{j}\left(i^{\prime}, j^{\prime}\right)+S_{j+3}\left(i^{\prime}, j^{\prime}\right.$ $+4)+S_{j+6}\left(i^{\prime}, j^{\prime}+8\right)+\ldots \quad\left[\right.$ similarly for $U_{j+1}\left(i^{\prime}, j^{\prime}\right)$ and $\left.U_{j+2}\left(i^{\prime}, j^{\prime}\right)\right]$ and then $U_{j}\left(i^{\prime}, j^{\prime}\right)+U_{j+1}\left(i^{\prime}, j^{\prime}+1\right)+U_{j+2}\left(i^{\prime}, j^{\prime}\right.$ $+2) \equiv U\left(i^{\prime}, j^{\prime}\right)$, we should arrive at a map in which the image of the three holes is visible. Moreover, the three holes in $U\left(i^{\prime}, j^{\prime}\right)$ should be at the same positions as those in $G_{i, j}\left(i^{\prime}, j^{\prime}\right)$.

We applied this protocol to $G_{i+\Delta i, j}\left(i^{\prime}, j^{\prime}\right)$ for $\Delta i=0,4,8$ starting from $i=30,31,32,33$ and, for each $i$, to $G_{i, j+\Delta j}\left(i^{\prime}, j^{\prime}\right)$ for $\Delta j=0,3,6$ starting from $j=57,58,59$. To calculate these 108 maps of correlation coefficients, $G_{i, j}\left(i^{\prime}, j^{\prime}\right)$, we performed the averaging operation of Eq. (1) on an ensemble of nine recorded intensity maps in that $108 \times 9$ is approximately equal to the number of records, namely 1000 , we used to calculate all previously shown correlation maps, including the one, $G_{34,61}\left(i^{\prime}, j^{\prime}\right)$ of Fig. 2(ii), that we replot in Fig. 5(a) as typical. We obtained the $U\left(i^{\prime}, j^{\prime}\right)$ that is plotted in Fig. 5 (b), in which the three holes appear at the expected positions. Such an image recovery is not due to an increase in the correlation coefficients, which are lower in (b) with respect to (a). It is rather attributable to the smearing out of the noise in the background. The shifts operated as described in agreement with the relations between the angles actually play a key role for obtaining a recovered image such as that in Fig. 5 (b): if the procedure leading from the $G_{i, j}\left(i^{\prime}, j^{\prime}\right)$ maps to $U\left(i^{\prime}, j^{\prime}\right)$ is applied without shifts, that is, if the 108 maps are simply summed pixel by pixel, we obtain the map shown in 
(a)

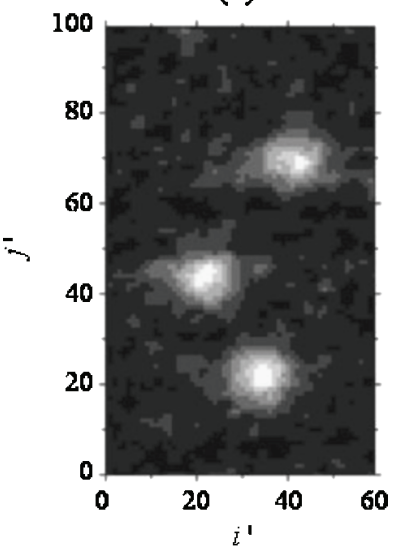

(c)

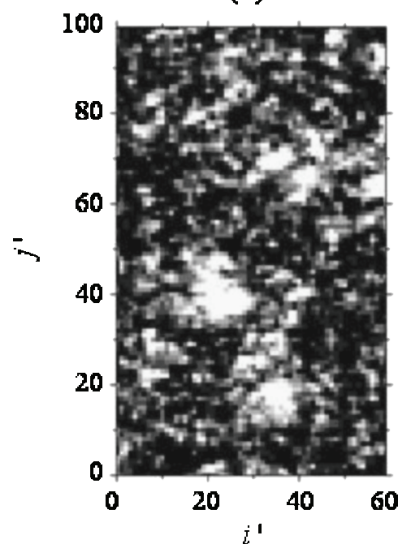

(b)

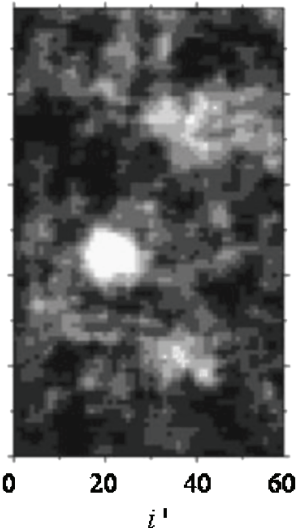

(d)

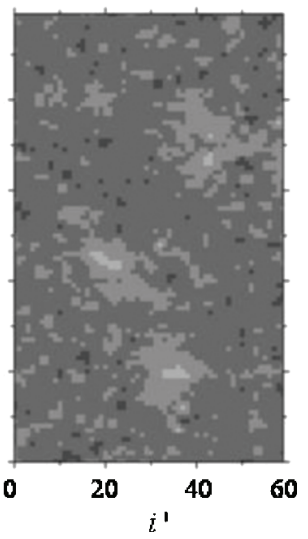

FIG. 5. (a) Enlarged view of the spatial intensity correlation map, $G_{34,61}\left(i^{\prime}, j^{\prime}\right)$, of Fig. 2(ii). (b) Map of the $U\left(i^{\prime}, j^{\prime}\right)$ matrix calculated by using an ensemble of only nine recorded intensity maps and the shift-and-add procedure. (c) Map of the matrix calculated as in (b) without shifts. The linear eight-level gray scale covers the same range of values in (b) as in (c). (d) Map of the correlation coefficients $G_{37,58}\left(i^{\prime}, j^{\prime}\right)$ chosen among the 108 maps used to obtain the $U\left(i^{\prime}, j^{\prime}\right)$ matrix plotted in (b). Criterion for the choice: highest visibility of the holes. The gray scale of (d) covers a range whose mean value is equal to that of (b) divided by 108 . The range is $\sqrt{108}$ times narrower.

Fig. 5(c) in which the noise prevents image recovery. Note that the content in a pixel $\left(i^{\prime}, j^{\prime}\right)$ of any of the maps in Figs. 5(a)-5(c) comes from about 1000 experimental determinations of local intensity (values recorded by the CCD pixels). In Fig. 5(a) these values are the content of as many pixels in the same position, namely $(34,61)$, of the 1000 recorded intensity maps $I_{2}\left(i^{\prime}, j^{\prime}\right)$; in the others they come from 108 correlation maps calculated by averaging over nine recorded intensity maps. The correlation coefficients were suitably shifted in position before summing to obtain Fig. 5(b), but were kept at the same positions to obtain Fig. 5(c). Finally, in Fig. 5(d), we show the $G_{i, j}\left(i^{\prime}, j^{\prime}\right)$ map out of the 108 used to obtain the results in Figs. 5(b) and 5(c), in which the three holes are most visible.

\section{APPLICATIONS}

We have demonstrated that a detailed knowledge of the rules set by PM on the recovery of our FDC images allows one to use a smaller number of recorded chaotic intensity maps for calculating the spatial intensity correlations in Eq. (1). If the randomization of the image is performed as a measure to impair visibility prior to transmission, this result could be exploited in a procedure for nonlocal image recovery.

As an extreme application of the rules in Eqs. (12) and (13), we point out that we could substitute the $N$ chaotic images to be used in Eq. (1) with as many shifted replicas of a single $I_{2}\left(i^{\prime}, j^{\prime}\right)$ map and nevertheless achieve image recovery. Actually, one could realize an experiment of image reconstruction in the limit case that only one chaotic intensity map $I_{2}\left(i^{\prime}, j^{\prime}\right)$ and the corresponding $I_{1}(i, j)$ be available, if the pixels are sorted as shown below to calculate

$$
\begin{aligned}
G_{i, j}\left(i^{\prime}, j^{\prime}\right)= & \frac{1}{N} \sum_{m=0}^{m_{\mathrm{MAX}}} \sum_{n=0}^{n_{\mathrm{MAX}}} I_{1}(i-m, j-n) I_{2}\left(i^{\prime}+m, j^{\prime}+n\right) \\
& -\frac{1}{N^{2}}\left[\sum_{m=0}^{m_{\mathrm{MAX}}} \sum_{n=0}^{n_{\mathrm{MAX}}} I_{1}(i-m, j-n)\right] \\
& \times\left[\sum_{m=0}^{m_{\mathrm{MAX}}} \sum_{n=0}^{n_{\mathrm{MAX}}} I_{2}\left(i^{\prime}+m, j^{\prime}+n\right)\right]
\end{aligned}
$$

in which $N=m_{\mathrm{MAX}} n_{\mathrm{MAX}}$. Note that this calculation is equivalent to that in Eq. (1) in that the statistical ensemble we consider here to calculate the correlation coefficients of Eq. (1) is given by the $N$ shifted maps, $I_{1}(i-m, j-n)$ and $I_{2}\left(i^{\prime}\right.$ $\left.+m, j^{\prime}+n\right)$, which are at most shifted by $m_{\mathrm{MAX}}$ and $n_{\mathrm{MAX}}$. In our case, for the sake of comparison with the results in Figs. 5 (a) and 5(b), we should cover up to $N \cong 1000$ when calculating the sums in Eq. (14).

Since with our setup the PM bandwidth is not as broad as it should be to allow light detection in so many pixels on plane $\mathrm{P}_{\mathrm{FT}}$, we present a demonstration based on a Monte Carlo numerical simulation. We constructed a $75 \times 75$ matrix of complex numbers with random real and imaginary parts (two Gaussian distributions with equal standard deviations and zero mean values) to be used as the Fourier components, $E_{1}(i, j)$, of the chaotic low-frequency input field. We calculated the $I_{1}(i, j)$ intensity map displayed in Fig. 6(a) as $\left|E_{1}(i, j)\right|^{2}$. We generated a two-level object mask in which the transparent holes were three squares of two-pixel sides located as in the real mask of Fig. 1(a). This "transparency" was used to calculate the transmitted portion of each field component [complex value of $E_{1}$ "recorded" at pixel $\left.(i, j)\right]$. All these maps of transmitted fields were displaced relative one to each other according to $\Delta i^{\prime}=-\Delta i$ and $\Delta j^{\prime}=-\Delta j$ and the pixel contents were summed afterwards to simulate the field impinging on the $\mathrm{P}_{\mathrm{IM}}$ plane. The squared magnitudes of the cell values of the resulting matrix, which are plotted in Fig. 6(b), were then used as $I_{2}\left(i^{\prime}, j^{\prime}\right)$ to calculate $G_{i, j}\left(i^{\prime}, j^{\prime}\right)$ according to Eq. (1), that is Eq. (14). As shown in Fig. 6(c) the $G_{i, j}\left(i^{\prime}, j^{\prime}\right)$ map satisfactorily recovers the original image, which is displayed in Fig. 6(d). 
(a)

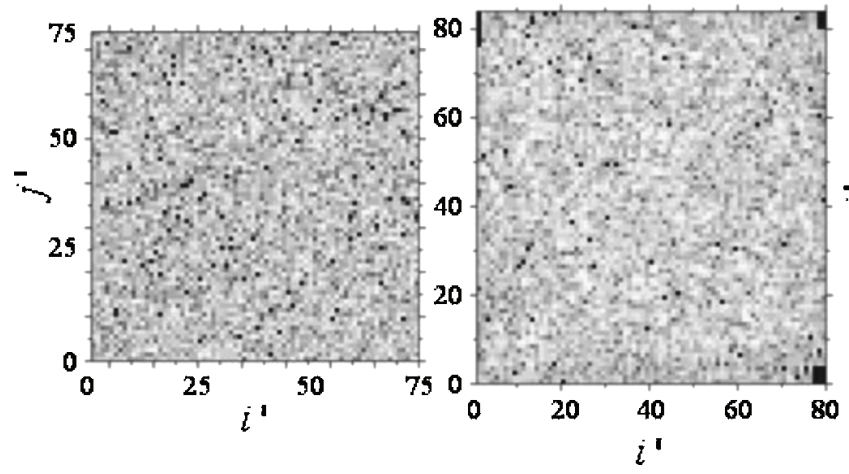

\section{CONCLUSIONS}

We have shown that the wave vectors in Eq. (9) fulfilling $\mathrm{PM}$ condition in 3D are linked to each other by rules that we exploited to devise a procedure to retrieve the FDC image from a limited number of records of chaotic FDC images. The method is based on the calculation of the correlations of the fluctuations of the local intensity of a set of chaotic images with those of the spatial Fourier components of the field used as the low-frequency input field in the crystal performing the downconversion process. Correlating with different Fourier components the same set of chaotic images and knowing the relative positions at which each correlation reconstructs the image can lead to successful retrieval even in the limit case that a single chaotic image (and spatial spectrum) is available, as we have shown by the numerical simulation in the last section. In the experiments we demonstrated the advantages of the procedure for a number of records (c)

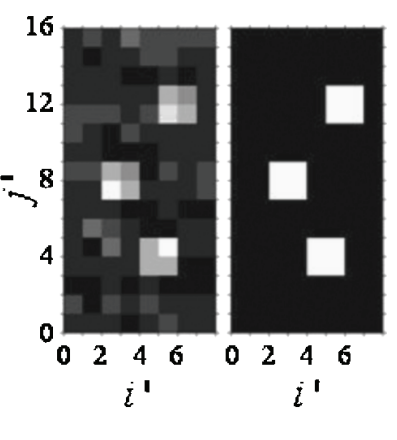

FIG. 6. Simulation of image recovery by using a single "recorded" FDC chaotic image, $I_{2}\left(i^{\prime}, j^{\prime}\right)$. (a) Map of $I_{1}\left(i^{\prime}, j^{\prime}\right)$ of the chaotic low-frequency input field. (b) Map of $I_{2}\left(i^{\prime}, j^{\prime}\right)$, see text. (c) Map of the correlation coefficients $G_{i, j}\left(i^{\prime}, j^{\prime}\right)$ calculated by the shift-and-add procedure as specified in Eq. (14). (d) "Object mask" used to calculate $I_{2}\left(i^{\prime}, j^{\prime}\right)$ for the field in (a).

greater than one. With our procedure, whichever is the number $N$ of recorded images and spectra, shifting and adding the maps of correlation coefficients calculated for different components is always convenient: the signal-to-noise ratio increases as the background noise smears out, though the number of data is still $N$. Finally we note that the procedure can be applied not only to FDC images but to any case in which recording the chaotic (noise-disturbed) images can be accompanied by the acquisition of the spatial Fourier spectrum of a field correlated to that producing the image.

\section{ACKNOWLEDGMENTS}

This work was partially supported by INFM through Project "PRA CLON" and by the Italian Ministry for University Research through the FIRB Project No. RBAU014CLC_002.

[1] R. H. T. Bates and F. M. Cady, Opt. Commun. 32, 365 (1980).

[2] Y. Sudo and N. Baba, Opt. Lett. 30, 1309 (2005).

[3] M. D'Angelo and Y. H. Shih, Laser Phys. Lett. 2, 567 (2005).

[4] D. Zhang, Y. H. Zhai, and L. A. Wu, Opt. Lett. 30, 2354 (2005).

[5] E. Puddu, A. Allevi, A. Andreoni, and M. Bondani, Opt. Lett. 30, 1294 (2005).

[6] F. Ferri, D. Magatti, A. Gatti, M. Bache, E. Brambilla, and L. A. Lugiato, Phys. Rev. Lett. 94, 183602 (2005).

[7] A. F. Abouraddy, B. E. A. Saleh, A. V. Sergienko, and M. C. Teich, J. Opt. Soc. Am. B 19, 1174 (2001).

[8] B. R. Mollow and R. J. Glauber, Phys. Rev. 160, 1076 (1967).

[9] E. Puddu, I. Degiovanni, A. Andreoni, M. Bondani, and S.
Castelletto, e-print quant-ph/0604143 (2006).

[10] F. T. Arecchi, Phys. Rev. Lett. 15, 912 (1965).

[11] M. Bondani, E. Puddu, and A. Andreoni, J. Mod. Opt. 53, 761 (2006).

[12] Bahaa E. A. Saleh and Malvin C. Teich, Fundamental of Photonics, 1st ed. (John Wiley \& Sons, New York, 1991), Chap. 6, p. 218.

[13] David N. Nikogosyan, Nonlinear Optical Crystals: A Complete Survey, 1st ed. (Springer, New York, 2005), Chap. 2, p. 5.

[14] A. Andreoni, E. Puddu, and M. Bondani, Appl. Phys. B: Lasers Opt. 85, 125 (2006).

[15] M. Bondani and A. Andreoni, Phys. Rev. A 66, 033805 (2002). 